\title{
ROTORDYNAMIC FORCES ACTING ON A CENTRIFUGAL OPEN IMPELLER IN WHIRLING MOTION BY USING ACTIVE MAGNETIC BEARING
}

\author{
N. Nagao ${ }^{1}$, M. Eguchi ${ }^{2}$, M. Uchiumi ${ }^{1}$, and Y. Yoshida ${ }^{1}$ \\ ${ }^{1}$ Japan Aerospace Exploration Agency \\ 1 Takakuzo, Jinjiro, Kakuda, Miyagi 981-1526, Japan \\ ${ }^{2}$ EBARA Corporation \\ 78-1 Shintomi, Huttsu, Chiba 293-0011, Japan
}

Rotordynamic forces acting on a centrifugal open impeller of a rocket engine turbopump were measured using a rotordynamic test stand controlled by active magnetic bearings. The tangential rotordynamic force $f_{t}$ had a small constantly negative value in the measured range. The direct stiffness $K$ had a positive value under various test conditions. In general, direct stiffness $K$ of a closed impeller had a negative value because of the Bernoulli effect. In the case of open impellers, the Bernoulli effect is speculated to be smaller because the absence of a front shroud makes $K$ positive.

\section{NOMENCLATURE}

$\begin{array}{ll}b_{2} & \text { outlet blade height of the impeller } \\ C & \text { dimensionless direct damping } \\ c & \text { dimensionless cross-coupled damping } \\ F_{n} & \text { normal rotordynamic force } \\ f_{n} & \text { dimensionless normal rotordynamic force } \\ F_{t} & \text { tangential rotordynamic force } \\ f_{t} & \text { dimensionless tangential rotordynamic force } \\ K & \text { dimensionless direct stiffness } \\ k & \text { dimensionless cross-coupled stiffness } \\ M & \text { dimensionless direct added mass } \\ m & \text { dimensionless cross-coupled added mass } \\ N=\Omega /(2 \pi) & \text { rotational frequency of the shaft } \\ Q & \text { volumetric flow rate } \\ Q_{n} & \text { reference volumetric flow rate }\end{array}$

This is an Open Access article distributed under the terms of the Creative Commons Attribution License 2.0, which permits unrestricted use, distribution, and reproduction in any medium, provided the original work is properly cited. 


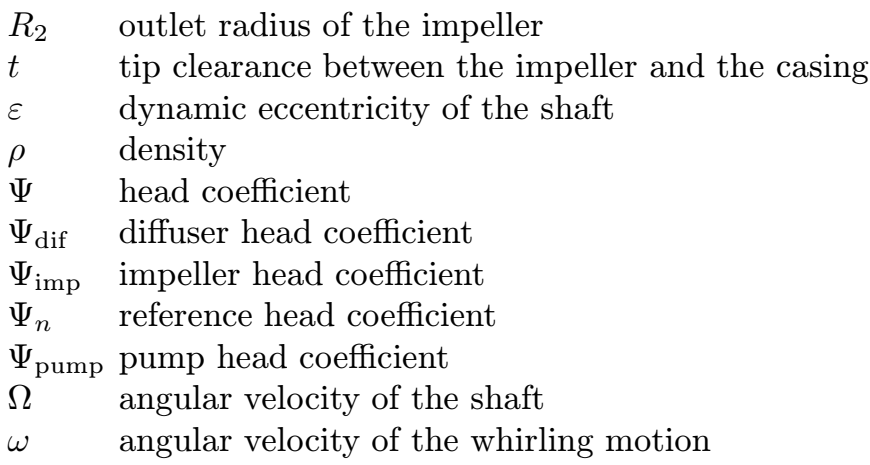

\section{INTRODUCTION}

For rocket engine turbopumps, not only a higher hydraulic performance but also a rotational stability are required. The energy density of rocket engine turbopumps is much higher than that of industrial turbopumps and they often operate at higher speed than the first critical speed of the rotor. Therefore, the problems caused by self-excited vibrations often occur. These vibrations are associated with the rotordynamic forces induced by the whirling motion of the rotor. To suppress the self-excited vibrations and keep the operation stable, it is important to clarify the rotordynamic forces acting on turbopump components and to apply that knowledge to the design of a turbopump system.

The rotordynamic forces acting on impellers have been studied since the development of the high-pressure fuel turbopump of the Space Shuttle Main Engine (SSME) [1]. The rotordynamic forces acting on "closed" impellers have been widely reported, and it is well known that they encourage instability in the low flow rate region [2]. On the other hand, there have been few reports on the rotordynamic forces acting in "open" impellers. In industrial open centrifugal impellers, it has been reported that rotordynamic forces encourage instability [3, 4]. The present study focuses on the rotordynamic forces acting on the "open" impeller in a rocket engine turbopump. The rotordynamic forces were measured by an experimental apparatus controlled by active magnetic bearings.

\section{EXPERIMENTAL APPARATUS}

The experiment was conducted by the use of the EBARA rotordynamic test stand (EBARTS) [5]. Figure 1 shows a cross-sectional view of the apparatus installed in the experimental open impeller. This apparatus is installed vertically. The shaft is rotated by an induction motor connected by a flexible coupling. 


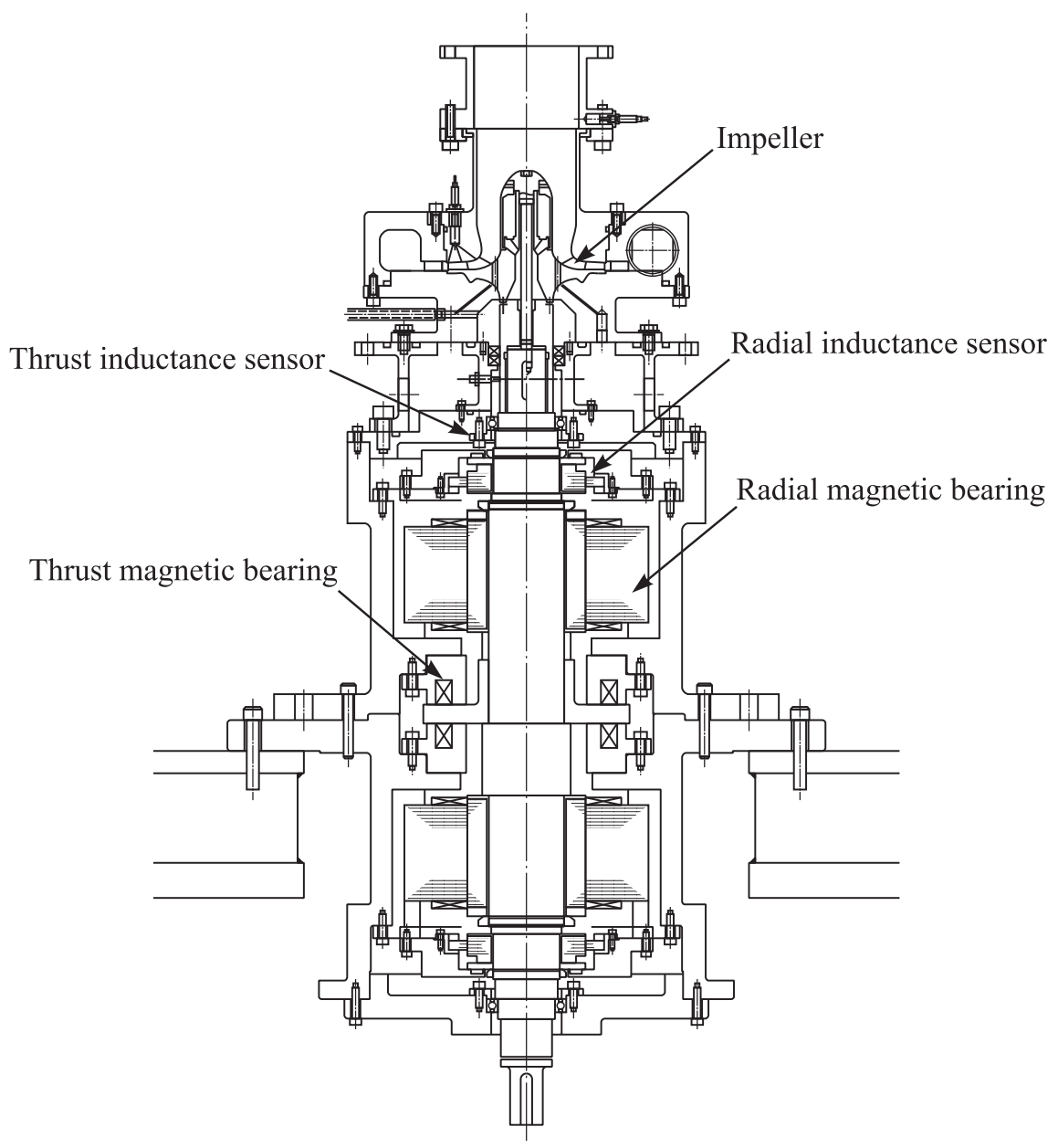

Figure 1 Cross-sectional view of the experimental apparatus

The radial and axial magnetic bearings control the rotor in 5 degree-of-freedom with the exception of the shaft rotational direction. This makes it possible to create measurement conditions under a noncontact state by levitating the rotor. Therefore, the rotordynamic forces can be measured with a high accuracy because of the low influence by other components. The rotordynamic forces can be evaluated by calculating the electromagnetic force from the control current of magnetic bearings.

Figure 2 shows the experimental centrifugal open impeller of a fuel turbopump for a rocket engine. The type number of this impeller is 0.63. 


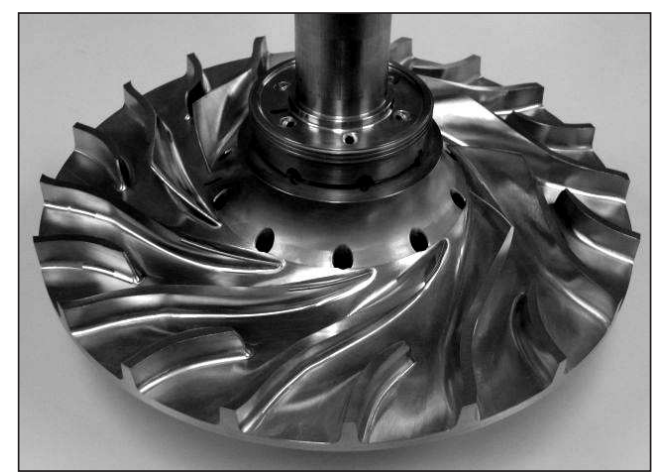

Figure 2 Experimental impeller

\section{DEFINITIONS}

Figure 3 shows a schematic of the rotordynamic forces acting on an impeller (the angular velocity of the shaft, $\Omega$ ) in whirling motion. The rotordynamic forces acting on the impeller caused by imposed whirling motion (the angular velocity of the whirling motion, $\omega$, and the dynamic eccentricity of the shaft, $\varepsilon)$ are decomposed into a force normal to the direction of the whirl motion $F_{n}$, and a force in the direction of the forward whirl motion $F_{t}$. These two forces are generally presented in dimensionless form as functions of the whirl frequency ratio $\omega / \Omega$ as follows:

$$
\begin{aligned}
& f_{n}=M\left(\frac{\omega}{\Omega}\right)^{2}-c \frac{\omega}{\Omega}-K \\
& f_{t}=-m\left(\frac{\omega}{\Omega}\right)^{2}-C \frac{\omega}{\Omega}+k
\end{aligned}
$$

Rotordynamic force effect at $\omega / \Omega>0$

$F_{n}>0$ : inertia effect

$F_{n}<0$ : restoring effect

$F_{t}>0$ : destabilizing effect

$F_{t}<0$ : damping effect

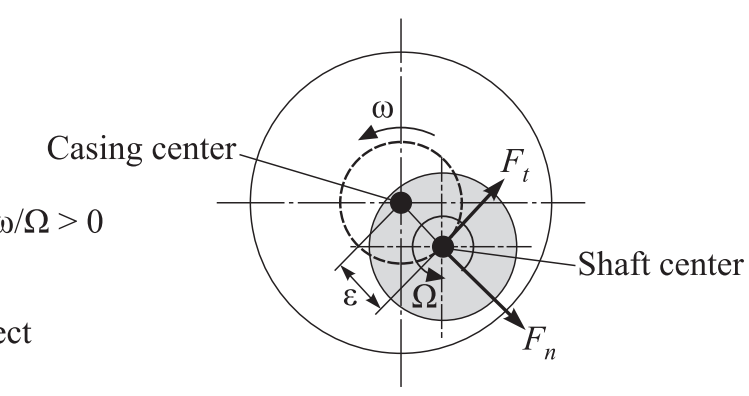

Figure 3 Schematic of the rotordynamic forces 
with forces normalized as follows:

$$
f_{n, t}=\frac{F_{n, t}}{\rho \pi R_{2}^{2} b_{2} \varepsilon \Omega^{2}}
$$

where $\rho$ is the fluid density; $R_{2}$ is the outlet radius of the impeller; $b_{2}$ is the outlet blade height of the impeller; and $M, m, C, c, K$, and $k$ are the rotordynamic coefficients.

In the range of $\omega / \Omega>0$, a positive tangential fluid force $\left(F_{t}>0\right)$ has a destabilizing effect because it promotes the whirl motion of the impeller. In the same way, a positive normal fluid force $\left(F_{n}>0\right)$ is destabilizing, but in a general turbopump, the radial stiffness of bearings is sufficiently large and thus does not negatively affect the turbopump system.

\section{RESULTS AND DISCUSSION}

\subsection{Performance Curve}

Figure 4 shows the performance curve at the dynamic eccentricity $\varepsilon=0$ and the rotational frequency $N=20 \mathrm{~Hz}$. The vertical axis is the head coefficient normalized by the reference head coefficient when $Q=Q_{n}$, and the horizontal axis is the volumetric flow rate normalized by the reference volumetric flow rate $Q_{n}$. The coefficient $\Psi_{\text {pump }}$ is defined by the difference between the pressure at the impeller inlet and the diffuser outlet, $\Psi_{\text {imp }}$ - between the impeller inlet and the impeller outlet, and $\Psi_{\text {dif }}$ - between the diffuser inlet and the diffuser outlet.

The coefficient $\Psi_{\text {pump }}$ constantly decreases in $Q / Q_{n}>0.5$. There is an inflection point in $Q / Q_{n}=0.3$. This point seems to be caused by a rotating stall.

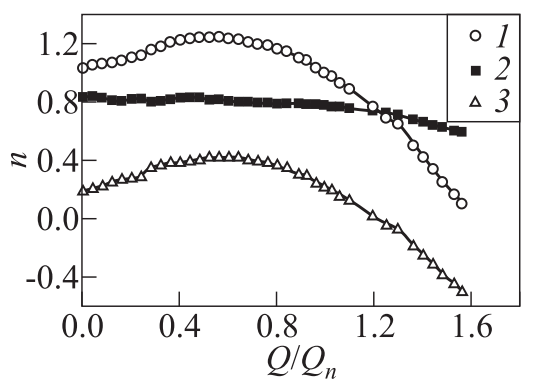

Figure 4 Performance curves of the experimental open impeller: $1-\Psi_{\text {pump }} / \Psi_{n}$; $2-\Psi_{\mathrm{imp}} / \Psi_{n} ;$ and $3-\Psi_{\mathrm{dif}} / \Psi_{n}$ 
Dividing $\Psi_{\text {pump }}$ into $\Psi_{\text {imp }}$ and $\Psi_{\text {dif }}$, it is seen that $\Psi_{\text {imp }}$ constantly decreases in all regions, on the other hand, $\Psi_{\text {dif }}$ increases in $0<Q / Q_{n}<0.6$ and is intensively disturbed in $Q / Q_{n}=0.3$. Therefore, this rotating stall is thought to occur in the diffuser. It is very interesting to evaluate the rotordynamic forces under the condition of the rotating stall; however, the rotordynamic forces in $Q / Q_{n}>0.6$ were focused on in this report.

\subsection{Rotordynamic Forces}

A series of tests has been conducted changing several parameters, namely, the volumetric flow rate ratio $Q / Q_{n}$, the dynamic eccentricity of the shaft $\varepsilon$, the rotational frequency of the shaft $N$, and the tip clearance $t$. The tip clearance indicates the axial distance between the impeller blade edge and the casing. Table 1 shows the matrix of the test condition. The values indicated by boldface are the nominal condition of each parameter.

Table 1 Test conditions

\begin{tabular}{cccc}
\hline $\begin{array}{c}\text { Flow rate } \\
\text { ratio } Q / Q_{n}\end{array}$ & $\begin{array}{c}\text { Dynamic eccentricity } \\
\text { of the shaft } \varepsilon, \mu \mathrm{m}\end{array}$ & $\begin{array}{c}\text { Rotational frequency } \\
\text { of the shaft } N, \mathrm{~Hz}\end{array}$ & $\begin{array}{c}\text { Tip clearance } t, \\
\mathrm{~mm}\end{array}$ \\
\hline $0.6,0.8, \mathbf{1 . 0}, 1.2$ & $100,150, \mathbf{2 0 0}$ & $15, \mathbf{2 0}$ & $0.4, \mathbf{0 . 5}, 0.6$ \\
\hline
\end{tabular}

Figures 5-8 show the experimental results of rotordynamic forces $\left(f_{n}\right.$ and $\left.f_{t}\right)$ under various test conditions. The figures on the left show $f_{n}$, and those of the right show $f_{t}$. The region $f_{t}>0$ at $\omega / \Omega>0$ (the shaded area) is the destabilizing area. Fitted quadratic curves (solid lines) are shown in these figures. Rotordynamic coefficients (described in the next section) are derived from these curves.
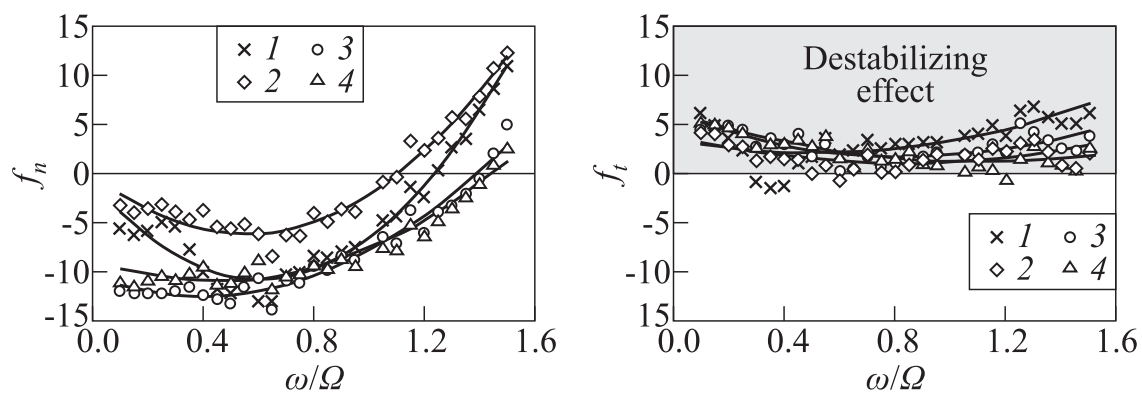

Figure 5 Comparison of rotordynamic forces at various volumetric flow rate ratios $Q / Q_{n}: 1-0.60 ; 2-0.80 ; 3-1.00 ;$ and $4-1.20$ 

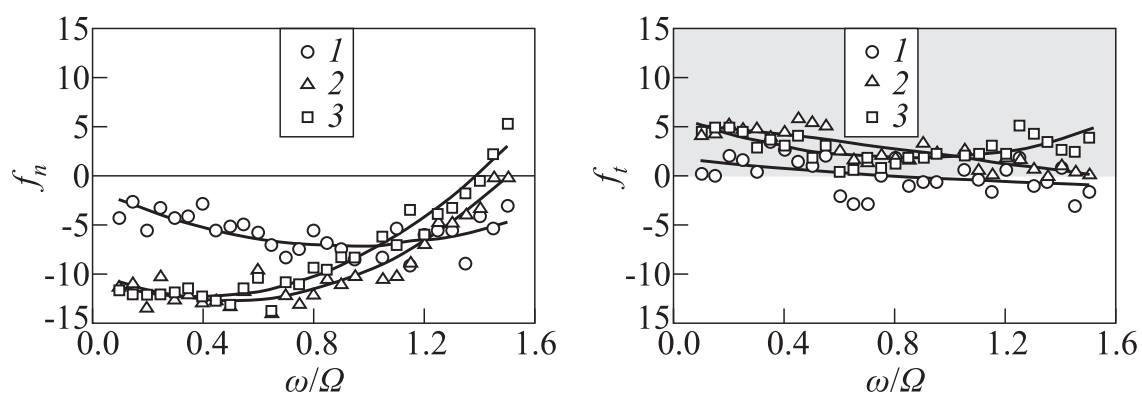

Figure 6 Comparison of rotordynamic forces at various dynamic eccentricities of the shaft $\varepsilon: 1-100 \mu \mathrm{m} ; 2-150$; and $3-200 \mu \mathrm{m}$
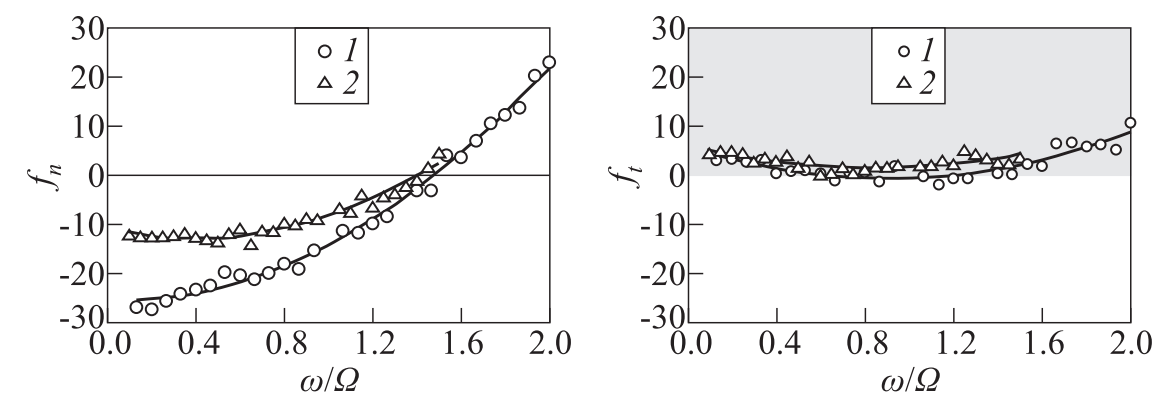

Figure 7 Comparison of rotordynamic forces at various rotational frequencies of the shaft $N: 1-15 \mathrm{~Hz}$; and $2-20 \mathrm{~Hz}$
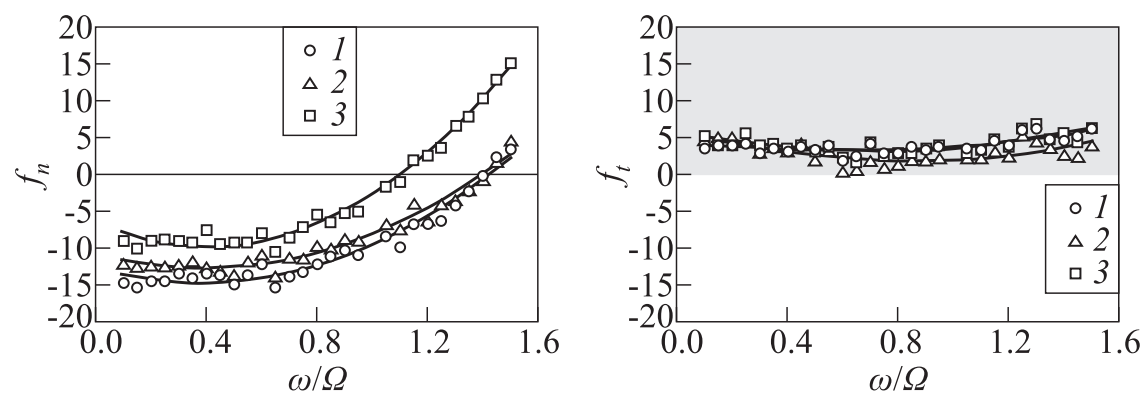

Figure 8 Comparison of rotordynamic forces at various tip clearances $t: 1-0.4 \mathrm{~mm}$; $2-0.5$; and $3-0.6 \mathrm{~mm}$ 
As a whole, both $f_{n}$ and $f_{t}$ acting on this open impeller are sufficiently small and stable. The force $f_{n}$ in this experiment is similar to the typical shape of $f_{n}$ acting on closed impellers. It has been previously reported [6] that for closed impellers, $f_{t}$ has a destabilizing effect in $0<\omega / \Omega<0.5$ because of interference with a volute casing or a vaned diffuser. By contrast, $f_{t}$ acting on this open impeller has a destabilizing effect in the whole measured range.

Figure 5 shows dimensionless rotordynamic forces at various volumetric flow rate ratios when the other parameters are nominal condition. The force $f_{t}$ is not affected by the flow rate. The force $f_{n}$ is relatively sensitive to the flow rate.

Figure 6 shows rotordynamic forces at various dynamic eccentricities of the shaft when the other parameters are nominal condition. The tendency of $f_{n}$ at $\varepsilon=100 \mu \mathrm{m}$ is different from those of the others. Namely, it is larger than the others in the low $\omega / \Omega$ and decreases with an increase of $\omega / \Omega$. The force $f_{t}$ at $\varepsilon=100 \mu \mathrm{m}$ sometimes has a negative value which means it does not have a destabilizing effect.

Figure 7 shows rotordynamic forces at various rotational frequencies of the shaft when the other parameters are nominal condition. The force $f_{t}$ is not much affected by the rotational frequency of the shaft. On the other hand, $f_{n}$ varies a great deal with the rotational frequency of the shaft. The force $f_{n}$ at $N=15 \mathrm{~Hz}$ is much smaller than at $N=20 \mathrm{~Hz}$ which means that $f_{n}$ at $N=15 \mathrm{~Hz}$ has a greater restoring effect.

Figure 8 shows rotordynamic forces at various tip clearances when the other parameters are nominal condition. The force $f_{t}$ is not affected by the tip clearance. The force $f_{n}$ decreases with tip clearance, which means that it has a greater restoring effect with a decrease of the tip clearance.

\subsection{Rotordynamic Coefficients}

Table 2 shows rotordynamic coefficients for various test conditions. They are derived from fitted quadratic curves of measured rotordynamic forces. From the test results it is noted that $K$ has a positive value, and $m$ is not negligibly small compared with $M$.

It has been reported that $K$ of closed impellers has a negative value [7] because of the Bernoulli effect [8]. In this experiment with the open impeller, $K$ has a positive value in almost all test conditions. Figure 9 shows the sensitivity of $K$ to various parameters. Figure $9 a$ shows that $K$ increases with the flow rate. Figure $9 b$ shows that $K$ increases with the dynamic eccentricity of the shaft. Figure $9 c$ shows that $K$ decreases with an increase of the rotational frequency of the shaft. Figure $9 d$ shows that $K$ decreases with an increase of the tip clearance. From Figs. $9 a$ and $9 c$, it seems that the larger the ratio of fluid velocity to the circumferential velocity of the impeller, the larger is the coefficient $K$. From Figs. $9 b$ and $9 d$, it seems that the smaller the clearance between 
Table 2 Rotordynamic coefficients

\begin{tabular}{cccccccccc}
\hline$N$ & $\varepsilon$ & $t$ & $Q / Q_{n}$ & $M$ & $m$ & \multicolumn{1}{c}{$C$} & $c$ & \multicolumn{1}{c}{$K$} & $k$ \\
\hline \multirow{2}{*}{1200} & \multirow{2}{*}{200} & \multirow{2}{*}{0.50} & 0.80 & 20 & -4.3 & 7.2 & 22 & -0.016 & 4.1 \\
& & & 1.00 & 13 & -6.2 & 10.0 & 9.9 & 11.0 & 6.1 \\
& & & 1.20 & 11 & -4.2 & 9.3 & 9.8 & 8.8 & 6.4 \\
\hline \multirow{2}{*}{1200} & \multirow{2}{*}{100} & \multirow{2}{*}{0.50} & \multirow{2}{*}{1.00} & 6.9 & -0.69 & 2.9 & 13 & 1.4 & 1.7 \\
& \multirow{2}{*}{150} & & & 13 & -0.098 & 4.0 & 13 & 9.6 & 5.8 \\
\hline \multirow{2}{*}{1200} & \multirow{2}{*}{200} & 0.40 & \multirow{2}{*}{1.00} & 14 & -3.6 & 4.3 & 11 & 12.0 & 4.4 \\
& & 0.60 & & 20 & -5.1 & 7.2 & 16 & 6.3 & 5.7 \\
\hline 900 & 200 & 0.50 & \multirow{2}{*}{1.00} & 12 & -7.9 & 14.0 & 1.0 & 25.0 & 6.0 \\
\hline
\end{tabular}

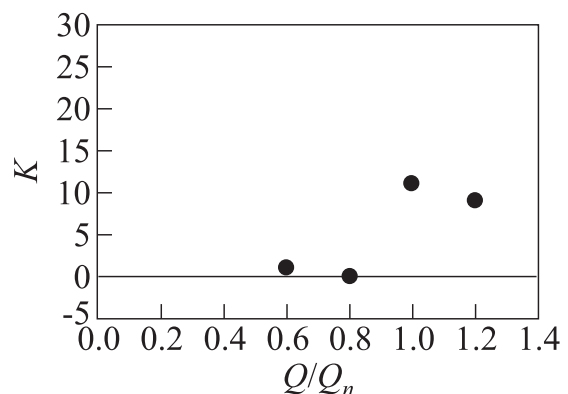

(a)

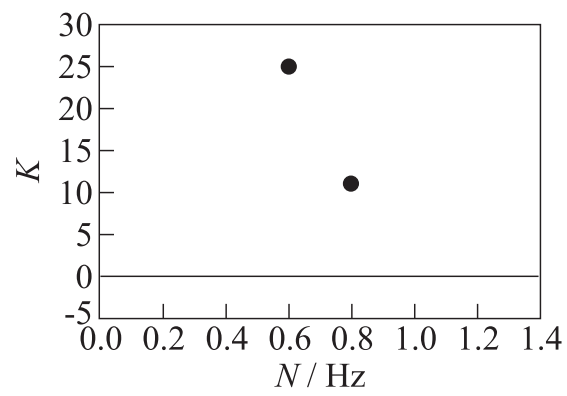

(c)

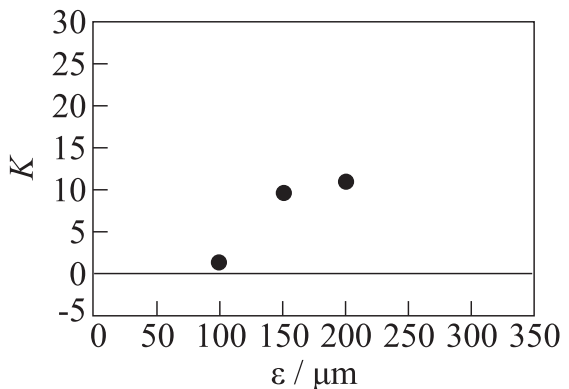

(b)

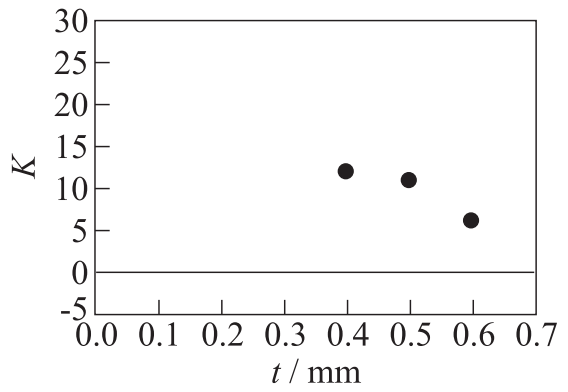

(d)

Figure 9 Sensitivity of $K$ to various parameters: $(a)$ to $Q / Q_{n} ;(b)$ to $\varepsilon ;(c)$ to $N$; and $(d)$ to $t$ 
the impeller blade edge and the casing, the larger is the coefficient $K$. It is noted that the tip clearance $t$ is the axial distance between the edge tip of the impeller blade edge and the casing; so, the radial tip clearance remains unchanged where the impeller and the casing are parallel. Therefore, the sensitivity to the tip clearance is relatively small.

These positive $K$ have been measured in some reports about rotordynamic forces acting on open impellers [9]. Here, this phenomenon is discussed in terms of the secondary flow velocity between the impeller and the casing. In a closed impeller, the discharge-to-suction leakage flow in the annulus surrounding the impeller shroud contributes substantially to the rotordynamic forces [10]. Adkins and Brennen reported that the leakage flow between the impeller shroud and the casing contributes to the normal rotordynamic force, which can be as much as $70 \%$ of the total [11]. In the case of impellers, the ratio of the axial length to the radius is so large that the Bernoulli effect (a negative stiffness) caused by the tangential bias of the tangential leakage flow velocity is larger than the Lomakin effect (a positive stiffness) caused by the tangential bias of the axial leakage flow velocity. Therefore, $K$ of closed impellers has a negative value. Open impellers do not have a front shroud, so it is thought that the tangential bias and the absolute value of the tangential velocity between the impeller and the casing are smaller than those of closed impellers. This small bias and absolute value of the tangential velocity makes the Bernoulli effect smaller; accordingly, $K$ becomes larger than that of closed impellers. This is similar to the swirl breaking effect. Previous experimental and analytical results have shown that $K$ of closed impellers increases with swirl brakes [12-14]. These experimental data show that swirl brakes increase the value of $K$.

In general, the absolute value of $m$ is negligibly small compared to $M$; so, $f_{t}$ is often described as a linear expression. In this experiment, however, $m$ is not negligibly small; so, $f_{t}$ needs to be fitted as quadratic curves. From equation (1), when the absolute value of $m$ is large, the minimum point of the $f_{t}$ quadratic curve has a positive value $\left(k+C^{2} /(4 m)>0\right)$. This contributes to $f_{t}$ having a positive value in the whole measured range $(0<\omega / \Omega<1.5)$. At $\varepsilon=100$ or $150 \mu \mathrm{m}, m$ is negligibly small. It is thought that $m$ increases with $\varepsilon$ and that there is a point where $m$ increases drastically between $\varepsilon=150$ and $200 \mu \mathrm{m}$. In actual operations, $\varepsilon$ is much smaller than $100 \mu \mathrm{m}$; so, it is thought that $f_{t}$ has a smaller destabilizing or damping effect.

\section{CONCLUDING REMARKS}

In this study, rotordynamic forces acting on the centrifugal open impeller were measured using a rotordynamic test stand controlled by active magnetic bearings under various test conditions. The relativity and sensitivity of measured 
rotordynamic forces and coefficients to the volumetric flow rate ratio $Q / Q_{n}$, the dynamic eccentricity of the shaft $\varepsilon$, the rotational frequency of the shaft $N$, and the tip clearance $t$ were evaluated. The experimental results and discussion can be summarized as follows:

(1) the change of the performance curve at $Q / Q_{n}=0.3$ seems to be caused by a diffuser rotating stall;

(2) the tangential rotordynamic force $f_{t}$ has a small positive value in the whole measured range, which means that it has a constant destabilizing effect;

(3) the direct stiffness $K$ has a positive value under almost all test conditions. There seems to be a smaller Bernoulli effect because the absence of a front shroud makes $K$ positive; and

(4) the cross-coupled added mass $m$ is not negligibly small compared with the direct added mass $M$. Thus, the tangential rotordynamic force $f_{t}$ has a positive value in the whole measured range.

\section{REFERENCES}

1. Ek, M. C. 1980. Sub-synchronous whirl in high-pressure turbomachinery. J. Spacecraft 17(3):208-18.

2. Ohashi, H., and H. Shoji. 1987. Lateral fluid forces on whirling centrifugal impeller. (2nd Report: Experiment in vaneless diffuser). ASME J. Fluids Eng. 109(2):100-6.

3. Yoshida, Y., Y. Tsujimoto, N. Ishii, H. Ohashi, and F. Kano. 1999. The rotordynamic forces on open-type centrifugal compressor impeller in whirling motion. ASME J. Fluids Eng. 121(2):259.

4. Hiwata, A., and Y. Tsujimoto. 2002. Theoretical analysis of rotordynamic fluid forces on an open-type centrifugal impeller in whirling motion. ASME J. Fluids Eng. 124:342.

5. Eguchi, M., and Y. Maruta. 2003. Development of rotordynamics measurement system with active magnetic bearings. 10th Asia-Pacific Vibration Conference Proceedings. Gold Coast, Australia. 1:115-20.

6. Jery, B., A. J. Acosta, C. E. Brennen, and T. K. Caughey. 1985. Forces on centrifugal pump impellers. 2nd Pump Symposium (International) Proceedings. Houston, Texas. 21-32.

7. Childs, D. 1993. Turbomachinery rotordynamics. New York: Wiley.

8. Brennen, C. E. 1994. Hydrodynamics of pumps. Oxford University Press.

9. Suzuki, T., R. Prunieres, H. Horiguchi, and Y. Tsujimoto. 2008. Experimental measurement of rotordynamic fluids forces on an open-type centrifugal impeller in whirling motion. ISROMAC12-2008-20131. 1-7. 
10. Adkins, D. R., and C. E. Brennen. 1988. Analyses of hydrodynamic radial forces on centrifugal pump impellers. ASME J. Fluids Eng. 110(1):20-28.

11. Jery, B., and R. Franz. 1982. Stiffness matrices for the rocketdyne diffuser volute. Calif. Inst. of Tech., Div. Eng. and Appl. Sci. Report No. E249.1.

12. Sivo, J. M., A. J. Acosta, C.E. Brennen, and T. K. Caughey. 1995. The influence of swirl brakes on the rotordynamic forces generated by discharge-to-suction leakage flows in centrifugal pumps. ASME 117:104-8.

13. Yun, H., and C.E. Brennen. 2002. Effect of swirl on rotordynamic forces caused by front shroud pump leakage. ASME J. Fluids Eng. 124:1005-10.

14. Brennen, C.E., and A. J. Acosta. 2005. Fluid-induced rotordynamic forces and instabilities. Structural Control Health Monitoring 13(1):10-26. 